Ann. Biol. anim. Bioch. Biophys., 1977, 17 (5 A), 679-694.

\title{
La gamétogenèse du mâle de Nectophrynoides occidentalis Angel (Amphibien Anoure vivipare). II. - Etude expérimentale du rôle des facteurs externes sur la spermatogenèse de l'adulte, au cours du cycle annuel
}

par Jacqueline GAVAUD

Laboratoire de Zoologie Ecole Normale Supérieure 46, rue d'UIm 75230 Paris Cedex 05.

Summary. Gametogenesis of the male Nectophrynoides occidentalis Angel (anurous viviparous amphibian). II. Experimental study of the role of external factors on adult spermatogenesis during the annual cycle.

The discontinuous spermatogenetic cycle of Nectophrynoides occidentalis evolves according to alternating dry and wet seasons. Under laboratory conditions we study the effect on spermatogenesis of external factors of the natural habitat : atmospheric humidity, frequency of feeding and day light ratio.

1) Force feeding and saturated atmospheric humidity at the beginning of underground life during the dry season induces spermatogenetic activity;

2) the onset of primary spermatogonia multiplication in January and that of secondary spermatogonia in April depend on internal factors ;

3) during the active period of the species in the rainy season, feeding factors cause significant changes in spermiogenesis. High saturation rate of atmospheric humidity and sufficient daylight ratio are also necessary to normal spermiogenesis.

\section{Introduction.}

Nectophrynoides occidentalis Angel, petit crapaud de l'Ouest Africain (Monts Nimba aux frontières de la Côte-d'lvoire, du Libéria et de la Guinée) possède un cycle écologique régi par l'alternance des saisons (voir fig. 4). Afin de se protéger de la sécheresse qui caractérise la période qui s'étend de novembre à fin mars, les $N$. occidentalis mènent une vie ralentie, cachés dans les fissures du sol ; ils perçoivent peu de lumière et les proies sont rares. L'émergence de la population n'a lieu qu'en avril et correspond au début de la saison des pluies. Jusqu'en octobre, la permanence des précipitations maintient le taux d'humidité de l'air proche de la saturation et les crapauds mènent une vie active à la surface du sol (Lamotte, 1959). Pendant la saison sèche, phase de vie souterraine de l'espèce, le cycle spermatogénétique est interrompu. L'activité gamétogénétique est alors limitée à la multiplication des spermatogonies I ef les testicules sont peu développés. A partir d'arril, la croissance testiculaire reprend et la suite de la 
spermatogenèse, de la multiplication des spermatogonies II à la spermiation se déroule en quatre mois (voir fig. 4). La spermiation commence fin juillet ef les accouplements ont lieu du mois d'août à la fin du mois d'octobre (Zuber-Vogeli et Xavier, 1965 ; Gavaud, 1975, 1976).

Fait exceptionnel pour une espèce tropicale, le cycle spermatogénétique est de type discontinu et la succession période de faible activité spermatogénétique-reprise de l'activité testiculaire ef spermiogenèse coïncide avec l'alternance saison sèchesaison humide (Angel et Lamotte, 1944, 1948 ; Lamotte, 1959 ; Zuber-Vogeli et Xavier, 1965 ; Gavaud, 1975, 1976). Quelle peut être alors l'influence des modifications saisonnières des facteurs physiques et nutritionnels du milieu sur le déterminisme du cycle spermatogénéfique de cette espèce?

\section{Matériel et méthodes.}

Techniques d'élevage au cours du cycle normal ef des expérimentations.

Les mâles, groupés par six dans de petits terrariums, sont élevés dans des chambres climatisées où les principaux facteurs physiques sont contrôlés (Gavaud, 1975).

1. Taux d'humidité atmosphérique. L'atmosphère peut être sèche (35 p. 100 d'humidité) ou presque saturée en humidité ( 90 p. 100).

2. Eclairement. Deux paramètres ont été considérés : l'intensité lumineuse (1000 lux, 50 lux, la semi-obscurité avec une intensité de 5 lux si la source lumineuse est artificielle ou en moyenne de 10 lux si la source lumineuse est naturelle et l'obscurité complète) et la photopériode (soit nulle, soit avec 12 h d'éclairement par 24 h programmées par une horloge).

3. Température. Aux Monts Nimba, les variations thermométriques sont amorties tout au long de l'année, à la surface comme à l'intérieur du sol, par un épais feutrage d'herbes. Le facteur température a donc été maintenu constant $\left(20 \pm 2{ }^{\circ} \mathrm{C}\right)$ au cours de l'ensemble des expérimentations.

4. Nourriture. Les mâles sont, selon les cas, alimentés tous les deux jours, tous les dix jours, ou tous les mois avec des drosophiles, des jeunes grillons et des vers de vase. La nourriture est pesée et les rations sont de :

- $25 \mathrm{mg}$ tous les deux jours ou tous les dix jours ;

- $50 \mathrm{mg}$ tous les mois.

Les rations alimentaires ont été établies d'après les résultats des études du bilan énergétique faites chez cette espèce (Lamotte, 1972).

Protocoles expérimentaux.

1. Rôle des facteurs externes sur la spermatogenèse au cours du cycle sexuel complet.

Deux séries d'expériences, portant sur 360 mâles, ont été réalisées au cours d'un cycle sexuel complet. Les crapauds, groupés par lots, sont soumis à différentes conditions physiques d'élevage. Les individus de chaque lot sont répartis en plusieurs groupes nourris à des fréquences différentes. Le tableau 1 donne toutes les combinaisons de 
Facteurs du milieu et spermatogenèse

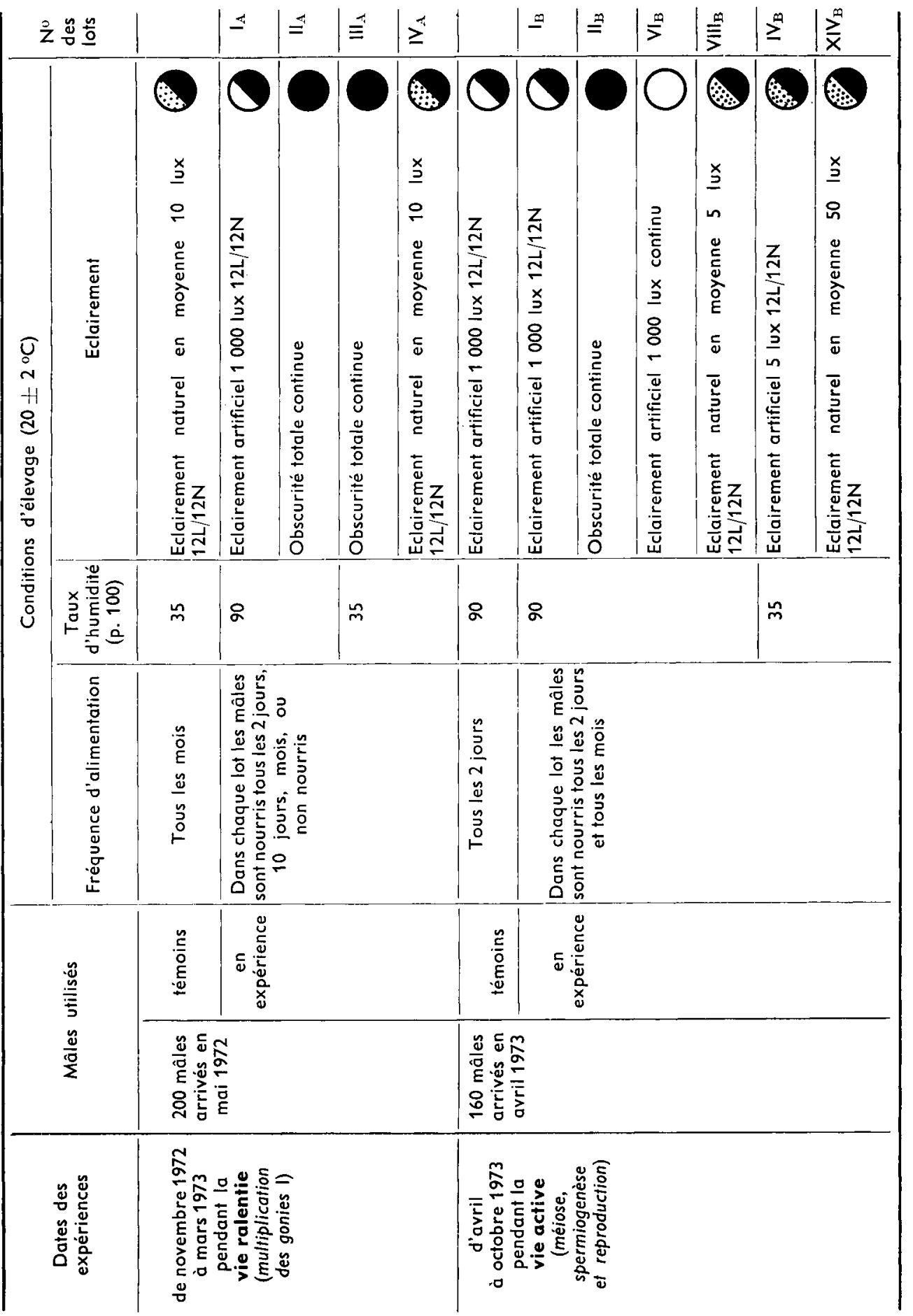


facteurs physiques (taux d'humidité et éclairement) réalisées et les conditions d'élevage des témoins. Tous les deux mois, les testicules de deux à trois mâles de chaque catégorie sont prélevés en vue d'une étude histologique et biométrique.

Lors de l'expérience menée pendant la phase de vie active de l'espèce, et dans le cas de mâles nourris tous les mois, seule l'autopsie de juillet a pu être faite : ce rythme d'alimentation au sortir de la période de vie ralentie s'est révélé insuffisant et a provoqué rapidement la mort des animaux en expérience.

2. Rôle des facteurs externes sur la spermatogenèse de mâles ayant subi une phase de vie ralentie anormale.

En avril, les mâles ayant survécu à la première expérimentation (menée de novembre à avril) ont été soit maintenus dans les mêmes conditions jusqu'en juin, soit soumis à des conditions différentes.

Techniques d'étude histologique et biométrique des festicules.

Après avoir été mesurés (du museau à la pointe de l'urostyle) et pesés, les crapauds sont tués au chloroforme et disséqués sous la loupe binoculaire. Les testicules sont pesés aussi rapidement que possible sur une balance électromagnétique Cahn (précise au $\mu \mathrm{g}$ ) puis fixés de 24 à 48 h au Bouin acétique. Ils sont inclus dans du « paraplast», coupés à $5 \mu$ et colorés à l'hémalun-éosine-bleu d'aniline ou à l'Azan. La spermiation est appréciée histologiquement.

L'influence des facteurs du milieu sur la spermatogenèse a été analysée quantitativement par la technique des numérations ponctuelles évaluant le pourcentage du volume du testicule occupé par chaque catégorie de cellule germinale (Solari, 1973 ; Billard et al., 1974 ; Gavaud, 1976). Cette technique nécessite l'emploi d'un oculaire intégrateur Zeiss I qui superpose à la coupe histologique une grille de points. Le dénombrement des points correspondant aux différents types de cellules permet d'évaluer le volume occupé :

$$
\frac{V \alpha}{V}=\frac{A \alpha}{A}=\frac{L \alpha}{L}
$$

V, A : volume et surface de la coupe; $L$ : nombre total de constituants de la coupe, soit 25 en utilisant l'oculaire intégrateur Zeiss; $V_{\alpha}, A \alpha$ : volume et surface d'un constituant ; $L \alpha:$ nombre de cellules de chaque catégorie.

\section{Résultats.}

\section{1. - Expérience menée pendant la période de vie ralentie de l'espèce}

$$
\text { (tabl. } 2 \text { et } 3 \text { ) }
$$

\section{A. Influence de la fréquence d'alimentation sur l'activité spermatogénétique.}

Lorsque les mâles sont alimentés tous les deux jours, quelles que soient les conditions physiques d'élevage, la multiplication des spermatogonies I est accélérée et se déroule en moins de deux mois ; la multiplication des spermatogonies II est déjà très avancée en janvier et la méiose commence (fig. 1). La structure du testicule n'est toutefois pas homogène : certains tubes séminifères ne contiennent que des spermatogonies I alors que d'autres sont en spermiogenèse. Cette hétérogénéité traduit l'aspect provoqué de la 
흠

\begin{tabular}{|c|c|c|c|c|c|c|c|c|c|c|c|}
\hline \multirow{4}{*}{ 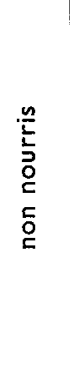 } & \multirow{2}{*}{$\begin{array}{l}\frac{8}{0} \\
\dot{0} \\
\text { ñ } \\
m\end{array}$} & & $\geq^{4}$ & & & & & & & & 1 \\
\hline & & & $\stackrel{\Xi}{\equiv}$ & $\begin{array}{ll}0 & 0 \\
& - \\
& -1\end{array}$ & & & & & & & 1 \\
\hline & \multirow{2}{*}{$\begin{array}{l}\frac{8}{0} \\
\dot{0} \\
\stackrel{2}{2}\end{array}$} & & $\underline{=}$ & $\dot{*}$ & $\bar{\sigma}^{\circ}+{ }_{1}$ & & & & & & $\stackrel{+}{+}$ \\
\hline & & & $=$ & $\begin{array}{l}0 \\
0 \\
\dot{0}+H\end{array}$ & & & & & & & 1 \\
\hline \multirow{4}{*}{ 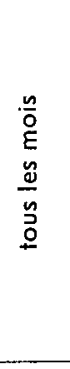 } & \multirow{2}{*}{$\begin{array}{l}\frac{8}{0} \\
\dot{\alpha} \\
\stackrel{n}{m}\end{array}$} & & $\geq$ & & & & & & & & + \\
\hline & & & 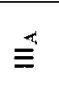 & $\begin{array}{ll}\stackrel{n}{n} & 0 \\
\dot{m} & = \\
m & =\end{array}$ & $\begin{array}{c}0 \\
0 \\
i \\
N\end{array}$ & $=\begin{array}{l}\approx \\
=\end{array}$ & & & & & + \\
\hline & \multirow{2}{*}{$\begin{array}{l}\frac{8}{0} \\
\dot{0} \\
\stackrel{2}{2}\end{array}$} & & $\stackrel{\pi}{=}$ & 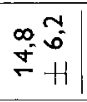 & 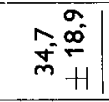 & $\left|\begin{array}{c}r \\
\hat{o} \\
0 \\
0 \\
+1\end{array}\right|$ & 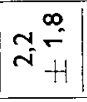 & $\left|\begin{array}{l}m_{m} \\
m^{2} \\
m^{\prime}\end{array}\right|$ & 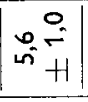 & $\stackrel{0}{\div}+$ & I \\
\hline & & & $\underline{I}$ & $\begin{array}{ll}0 \\
0 \\
i \\
i\end{array}$ & 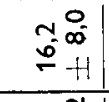 & 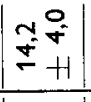 & & & & & 1 \\
\hline \multirow{4}{*}{ 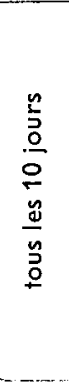 } & \multirow{2}{*}{$\begin{array}{l}\frac{8}{0} \\
\dot{0} \\
\dot{m} \\
m\end{array}$} & & $\geq$ & 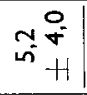 & 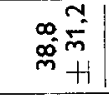 & & $\mid \begin{array}{ccc}2 & m \\
0 & + \\
0\end{array}$ & $\left|\begin{array}{l}n \bar{n} \\
\tilde{n}^{-}\end{array}\right|$ & $\left|\begin{array}{l}0 \\
0 \\
0 \\
-\end{array}\right|$ & $\underset{\infty}{n}+1$ & 1 \\
\hline & & & 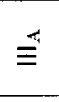 & 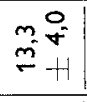 & 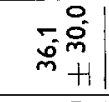 & & 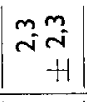 & & & & 1 \\
\hline & \multirow{2}{*}{$\begin{array}{l}\frac{8}{\circ} \\
\frac{0}{8}\end{array}$} & & $=$ & 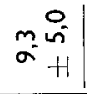 & 足 & $\left|\begin{array}{l}0 \\
0 \\
0 \\
-1 \\
1\end{array}\right|$ & $\left(\begin{array}{l}2 \\
= \\
-1\end{array}\right.$ & $\left|\begin{array}{l|}0 \\
\stackrel{0}{=} \\
-H\end{array}\right|$ & $\left|\begin{array}{l}\cong \\
\hdashline \\
-1\end{array}\right|$ & & 1 \\
\hline & & & $\leq$ & 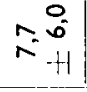 & 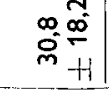 & $\left|\begin{array}{l}=0 \\
=0 \\
F\end{array}\right|$ & $\mid \begin{array}{l}0 \\
m \\
m \\
m \\
m\end{array}$ & $\stackrel{2}{-} \frac{a}{+}$ & $\left|\begin{array}{l}n \\
2 \\
-1 \\
+1\end{array}\right|$ & $\stackrel{00}{-1}$ & 1 \\
\hline \multirow{4}{*}{ 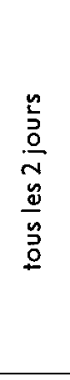 } & \multirow{2}{*}{$\begin{array}{l}\frac{8}{0} \\
\dot{0} \\
\dot{m} \\
m\end{array}$} & & $\geq$ & $\stackrel{0}{+}=$ & $\begin{array}{c}0 \\
\tilde{y} \\
\dot{y} \\
+H\end{array}$ & & 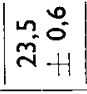 & $\left|\begin{array}{cc}n & n \\
0 & 0 \\
0 & H\end{array}\right|$ & & & I \\
\hline & & & $\equiv$ & 욤 & 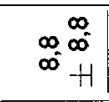 & & $\mid \begin{array}{r}\hat{0} \\
a^{2} \\
0 \\
0\end{array}$ & $\mid \begin{array}{ll}\infty & \infty \\
0 & 0 \\
0 & 0 \\
0\end{array}$ & 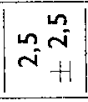 & $\begin{array}{l}0 \\
\text { o. } \\
\infty^{0} \\
n^{\circ}+1 \\
+1\end{array}$ & + \\
\hline & \multirow{2}{*}{$\begin{array}{l}\frac{8}{0} \\
\vdots \\
8\end{array}$} & & $=$ & $\begin{aligned} \forall & 0 \\
0 & \end{aligned}$ & 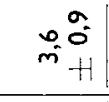 & & $\mid \begin{array}{c}2 \\
i \\
i\end{array}$ & $\left|\begin{array}{c}\frac{m}{\bar{N}} \\
\bar{n}\end{array}\right|$ & 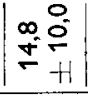 & $\begin{array}{l}\stackrel{0}{\circ} \\
\stackrel{m}{F}+H\end{array}$ & I \\
\hline & & & $\angle$ & \begin{tabular}{r}
$\infty$ \\
0 \\
\hdashline \\
- \\
+1
\end{tabular} & 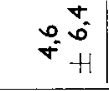 & $\hat{0}$ & 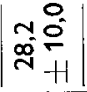 & 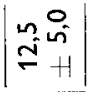 & $\mid \begin{array}{l}0 \\
0 \\
0 \\
0 \\
0 \\
-\infty\end{array}$ & $\frac{0}{-1}$ & 1 \\
\hline 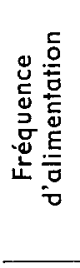 & 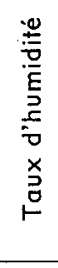 & 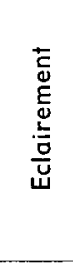 & 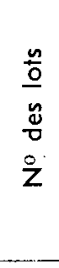 & 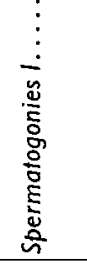 & 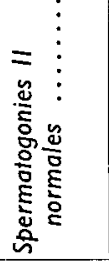 & 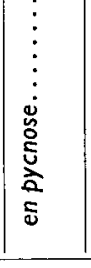 & 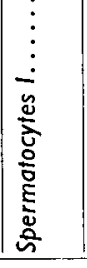 & 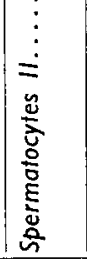 & 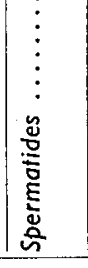 & 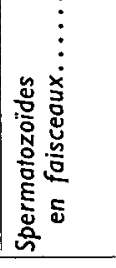 & \multirow[t]{2}{*}{ 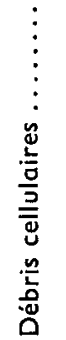 } \\
\hline & & & & & & & & & & & \\
\hline
\end{tabular}


spermatogenèse. Dans tous les lots, les spermatogonies il pycnotiques représentent de 10 à 20 p. 100 du volume de la gonade puis seulement 0,7 p. 100 deux mois plus tard. La suite du développement de la spermatogenèse semble nettement conditionnée par les facteurs physiques, taux d'humidité et éclairement.

Si la ration alimentaire n'est distribuée que fous les dix jours, l'accélération de la multiplication des spermatogonies 1 est encore nette, bien que moins accentuée que dans le groupe précédent; cette phase dure de trois à quatre mois. En revanche, ces animaux sont plus sensibles que les premiers aux conditions d'humidité et d'éclairement.

Une fréquence d'alimentation mensuelle ne provoque pas d'accélération de la multiplication des spermatogonies primaires, seuls l'hygrométrie de l'air et l'éclairement semblent modifier le déroulement de la spermatogenèse.

La dénutrition due au jeûne entraîne une activité spermatogénétique anormalement faible. La lumière des tubes séminifères est obstruée par des débris cellulaires et la taille des testicules est réduite (fig. 1).

B. Influence du taux d'humidité atmosphérique sur la spermatogenèse.

1. Mâles élevés en atmosphère humide.

Lorsque les mâles sont abondamment nourris (tous les 2 jours) pendant la période normale de jeûne de l'espèce, l'influence stimulante du taux élevé d'humidité atmosphé-

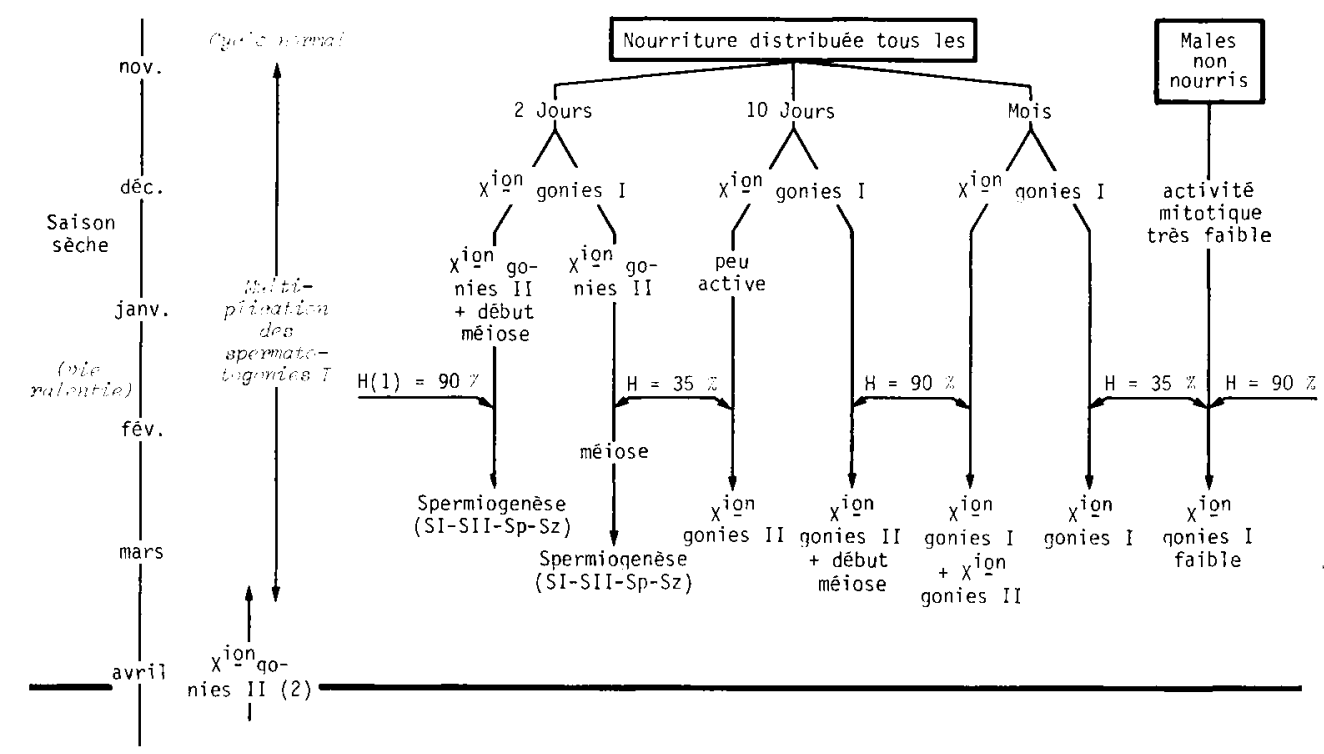

FIG. 1. - Rôle de la fréquence d'alimentation ef du taux d'humidité atmosphérique sur la spermatogenèse de N. occidentalis.

(1) $\mathrm{H}=$ taux d'humidité atmosphérique

(2) Xion $=$ multiplication. 
rique n'est vraiment nette qu'en mars alors que les mâles élevés soit à l'obscurité (Lot IIA) soit à la lumière pendant $12 \mathrm{~h}$ sur $24 \mathrm{~h}$ (Lot IA) sont en spermiation ; leurs testicules contiennent des faisceaux de spermatozoìdes constituant 5 à 20 p. 100 de la gonade, qui pèse alors entre 4 et $5 \mathrm{mg}$ (fig. 1 ) : le développement des festicules et de la spermatogenèse est alors identique à celui des animaux témoins autopsiés au mois de juillet.

Les mâles alimentés tous les dix jours s'avèrent plus sensibles que ceux du groupe précédent au taux d'humidité de l'air ainsi qu'à l'éclairement. Les animaux du lot IA, éclairés $12 \mathrm{~h}$ sur 24 ( $1000 \mathrm{lux}$ ) en milieu très humide, possèdent en janvier, des testicules en méiose. En mars, dans ce même groupe, la multiplication des spermatogonies II a encore lieu ; la faible fréquence d'alimentation limite le développement de la méiose observé en janvier. Dans les mêmes conditions d'humidité mais à l'obscurité, seule la multiplication des spermatogonies II est active (fig. 1).

L'association atmosphère humide-éclairement important tend à provoquer chez les animaux nourris tous les mois la multiplication accélérée des spermatogonies I et ll et même, dès janvier, la méiose. Toutefois ce développement précoce de la spermatogenèse n'évolue pas par la suite en raison de la sous-alimentation des mâles (fig. 1). Les gonades présentent quelques anomalies : cystes de spermatogonies II aux contours très imprécis ef pycnoses des spermatogonies $I I$ et $I$.

Les mâles non nourris peuvent présenter, en janvier, une légère stimulation de la spermatogenèse à condition qu'ils soient également soumis à un éclairement important (1000 lux, 12L/12N, lot IA ) : $40 \mathrm{p} .100$ du volume des testicules, alors très réduits, sont occupés par des spermatogonies II. En mars, l'activité spermatogénétique diminue fortement et devient plus faible qu'au cours du cycle normal à la même date.

\section{Mâles élevés en atmosphère sèche.}

Dans l'ensemble, l'atmosphère sèche ne favorise pas le développement de l'activité spermatogénétique.

Si la nourriture est distribuée tous les deux jours, quel que soit l'éclairement (lots IIIA et IVA) les testicules ne pèsent en moyenne que $2,5 \mathrm{mg}$ et la spermiogenèse est moins avancée que chez les mâles élevés en atmosphère humide (fig. 1).

$\mathrm{Si}$ les crapauds sont alimentés tous les dix jours, les résultats sont hétérogènes. Dans le cas d'un éclairement faible (lot IVA), la multiplication des spermatogonies I peu active en janvier est néanmoins achevée deux mois plus tard, en mars. La multiplication des spermatogonies II est alors intense et quelques cystes de spermatocytes et de spermatides sont déjà présents. En revanche, dans le cas des mâles élevés à l'obscurité complète, les divisions des spermatogonies I et II sont très fréquentes en janvier ; en mars la spermatogenèse n'a pas évolué davantage.

Le développement des festicules ef de la spermatogenèse des mâles alimentés une fois par mois, quel que soit l'éclairement, sont identiques à ceux des animaux témoins, aussi bien en janvier qu'en mars (fig. 1). Les phénomènes de dégénérescence cellulaire sont toutefois plus importants qu'au cours du cycle normal.

Si les mâles élevés dans de telles conditions d'humidité, quel que soit l'éclairement, sont maintenus en état de jeûne, l'activité spermatogénétique est bien moins intense qu'au cours du cycle normal (fig. 1). 


\section{Influence de l'éclairement sur l'activité spermatogénétique.}

L'éclairement ne semble pas particulièrement intervenir lorsqu'il s'agit d'animaux abondamment nourris. Dans tous les autres cas (jeûne ou nourriture donnée tous les dix jours ou tous les mois), l'association d'un éclairement de 1000 lux avec photopériode $12 \mathrm{~L} / 12 \mathrm{~N}$ et d'un taux d'humidité atmosphérique élevé stimule la spermatogenèse. En revanche, quel que soit le taux d'humidité ambiant, l'obscurité tend à freiner le développement de l'activité spermatogénétique.

\section{Expérience menée pendant la période de vie active de l'espèce}

A. Rôle des facteurs externes sur la spermatogenèse de mâles ayant subi une phase de vie ralentie normale (tabl. 4).

\section{Influence de la fréquence d'alimentation sur la spermatogenèse.}

Une sous-alimentation pendant la période normale de vie active de l'espèce provoque à elle seule un ralentissement prolongé de la spermiogenèse ; les facteurs physiques du milieu ne semblent pas produire de modifications particulières (fig. 2). En juillet, les testicules de ces mâles sont de taille réduite du fait de la dénutrition et contiennent des spermagotonies I, des cystes de spermatogonies II comprenant une fraction élevée de ccllules pycnotiques et des cystes de spermatocytes I dont les contours sont souvent mal définis. Par ailleurs, les tubes séminifères sont très souvent obstrués par des débris cellulaires. En août, seuls quelques individus des lots IB et VIIIB ont survécu et ont pu être étudiés. Lorsque les conditions d'élevage sont proches de celles du milieu naturel (lot lB) les testicules contiennent des spermatozoïdes épars alors que dans le cas des mâles du lot VIIIB ( 90 p. 100 d'humidité, semi-obscurité avec photopériode 12L/12N) la spermatogenèse ne dépasse pas le stade de la multiplication des spermatogonies II.

II n'est donc pas possible d'affirmer qu'une sous-alimentation pendant la période de vie active provoque systématiquement l'inhibition de la spermatogenèse. Les conditions d'humidité et d'éclairement semblent, dans ce cas, jouer un rôle important.

En revanche, la spermiogenèse des mâles bien alimentés aboutit, dans tous les cas, à la formation de spermatozoïdes et à la spermiation en août. Si le taux d'humidité de l'air et l'éclairement sont modifiés par rapport aux conditions normales de cette saison, des perturbations telles que des retards par rapport au cycle annuel et des pycnoses cellulaires apparaissent.

Une alimentation fréquente (tous les 2 jours) et des conditions d'humidité ( 90 p. 100) et d'éclairement ( 1000 lux avec photopériode $12 \mathrm{~L} / 12 \mathrm{~N}$ ) les plus proches des conditions naturelles pendant la saison humide (lot $I B$ ) provoquent une spermiogenèse normale sans dégénérescences cellulaires et dans les mêmes délais qu'au cours du cycle annuel (fig. 2). Toute modification de l'un ou l'autre de ces facteurs provoque soit des retards par rapport au déroulement normal soit des pycnoses qui n'empêchent en aucun cas la formation de spermatozoïdes et la spermiation (fig. 2). La seule perturbation ayant entraîné une diminution nette de la quantité de spermatozoìdes formés est la dégéné- 


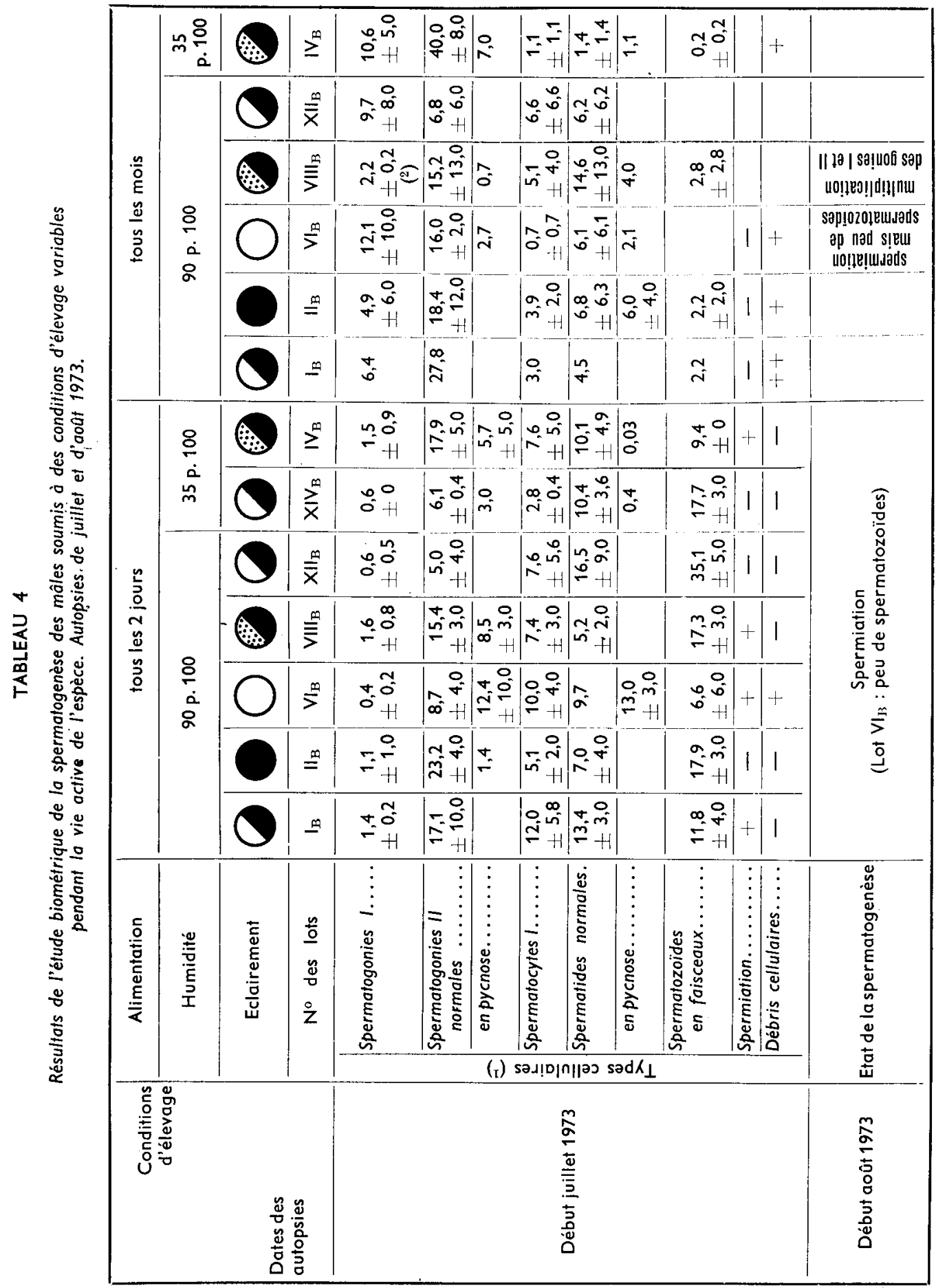


rescence d'une fraction importante de spermatides provoquée par l'exposition des mâles à un éclairement artificiel continu de 1000 lux (lot VIB).

Lorsque les animaux sont sous-alimentés, les facteurs humidité et éclairement ne semblent pas avoir d'influence significative.

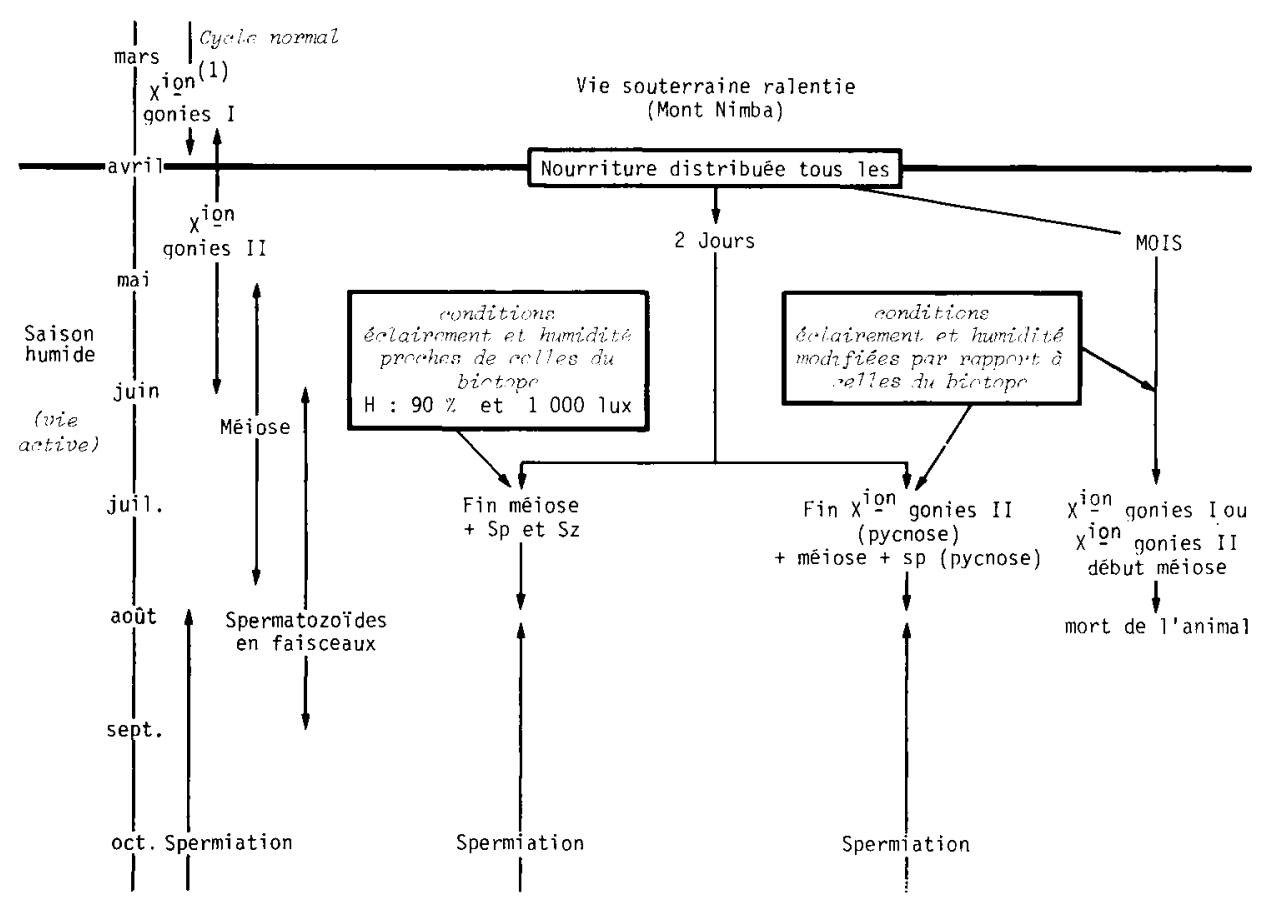

FIG. 2. - Rôle des facteurs externes sur la spermatogenèse de N. occidentalis en période de vie active : cas des mâles ayant subi une phase de vie ralentie normale.

(1) Xion $=$ multiplication

(2) $\mathrm{H}=$ taux d'humidité atmosphérique.

B. Rôle des facteurs externes sur la spermatogenèse de mâles ayant subi une phase de vie ralentie anormale (fig. 3).

Un premier groupe d'individus a été maintenu en état de vie active (olimentation tous les deux jours, humidité de 90 p. 100, éclairement de 1000 lux pendant 12 h) de novembre à avril puis d'avril à juin (fig. $3 a$ ). La spermatogenèse induite dès novembre avec la multiplication accélérée des gonies I, se poursuit normalement jusqu'à la spermiation avec trois mois d'avance. Un autre groupe est constitué de mâles en vie ralentie (alimentation tous les mois, humidité 35 p. 100 et semi-obscurité) depuis novembre (fig. $3 b$ ). En juin, la spermatogenèse semble avoir lieu malgré l'état de dénutrition des mâles : les trois testicules prélevés, pèsent en moyenne $0,3 \mathrm{mg}$ et contiennent quelques spermatozoïdes. 
D'autres $N$. occidentalis jeûnant depuis novembre, ont été élevés à partir d'avril dans les conditions de la vie active (fig. $3 c$ ). En août, leurs testicules ont pratiquement atteint un poids normal et les tubes séminifères sont comblés par des spermatozoïdes en cours d'évacuation, alors qu'en avril, la multiplication des spermatogonies primaires n'était pas commencée. Ceci implique que la multiplication des spermatogonies

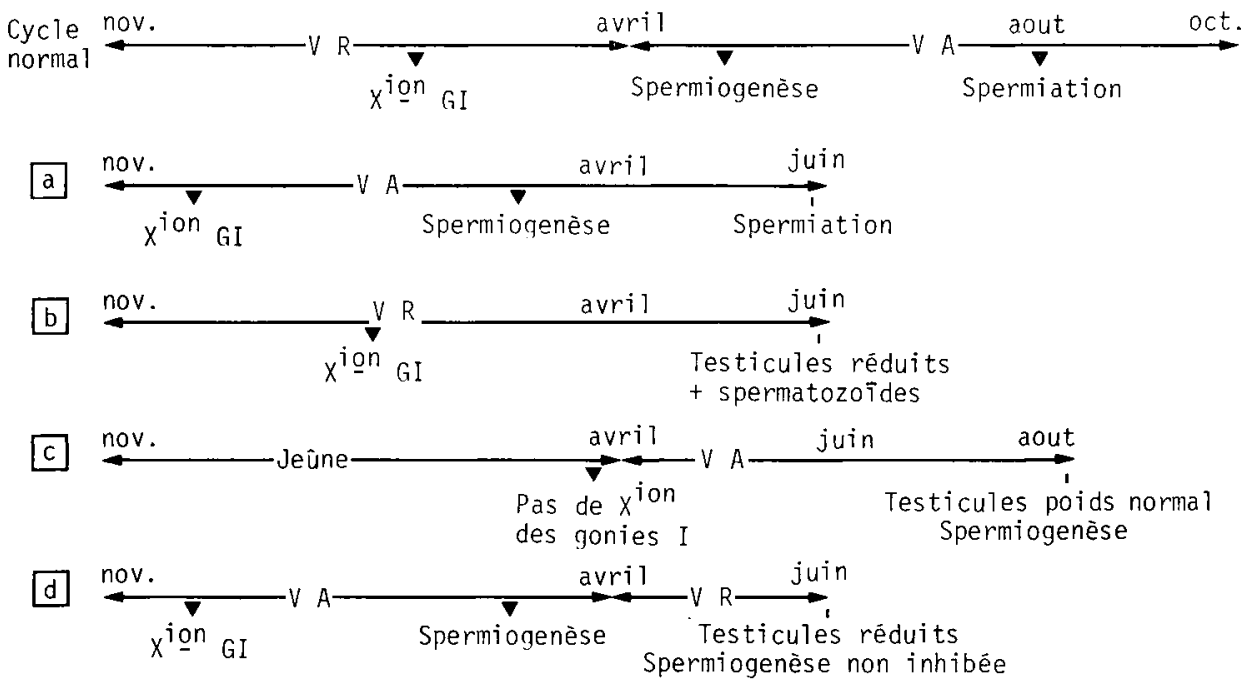

FIG. 3. - Rôle des facteurs externes sur la spermatogenèse de mâles n'ayant pas subi unéphaséde vie ralentie normale.

Xion $\mathrm{Gl}=$ multiplication des spermatogonies primaires

$V A=$ vie active $\left\{\begin{array}{l}\text { nourriture tous les deux jours } \\ \text { humidité } 90 \text { p. } 100 \\ \text { éclairement } 1000 \text { lux } 12 \mathrm{~L} / 12 \mathrm{~N}\end{array}\right.$

$V R=$ vie ralentie $\left\{\begin{array}{l}\text { nourriture tous les mois } \\ \text { humidité } 35 \text { p. } 100 \\ \text { semi-obscurité } 12 \mathrm{~L}-12 \mathrm{~N} .\end{array}\right.$

ef la spermiogenèse se soient déroulées en trois mois et demi au lieu de huit mois comme pendant le cycle normal. Si au contraire, des mâles en vie active de novembre à avril sont mis en état de vie ralentie, la spermiogenèse déjà bien avancée en avril, n'est pas inhibée par le passage à des conditions de vie défavorables (fig. 3d).

\section{Discussion.}

Les interactions de facteurs externes et de facteurs internes dans la régulation de la spermatogenèse des Amphibiens ont été mises en évidence dès les premiers travaux d'hypophysectomie (Bufo arenarum, Guisti et Houssay, 1923) et d'étude de l'intervention de facteurs climatiques sur la spermatogenèse (Rana temporaris, Witschi, 1924). Les recherches entreprises par la suite chez plusieurs espèces d'Amphibiens permettent de distinguer trois types de cycles spermatogénétiques : un cycle continu avec produc- 
tion de sperme toute l'année, observé surtout dans les zones tropicales et subtropicales, un cycle discontinu sensu stricto sous la seule dépendance d'un rythme interne, et enfin un cycle « potentiellement continu » caractéristique des Amphibiens des régions tempérées (Van Oordt, 1960). Pour ces espèces, ce sont les basses températures d'hiver qui induisent l'arrêt de la spermatogenèse (Rana esculenta, Galgano, 1935 ; Rana iberica et Rana graeca, Crespo et Cei, 1971). Ce facteur température peut également modifier la spermatogenèse de Batraciens dont le cycle discontinu est principalement réglé par un rythme interne (Rana temporaria, Van Oordt, 1956). La photopériode et l'intensité lumineuse interviennent peu dans le déroulement des cycles sexuels (Plethodon cinereus, Werner, 1969). Le jeûne comme la surabondance de nourriture perturbent chez les espèces étudiées la spermatogenèse sans jamais provoquer son inhibition.

L'originalité des mâles de Nectophrynoides occidentalis ne réside donc pas seulement dans le fait qu'ils possèdent, en zone tropicale, un cycle spermatogénétique discontinu, mais aussi que des facteurs externes autres que la température participent à la régulation du cycle sexuel : la fréquence d'alimentation, le taux d'humidité atmosphérique et, de façon moindre, l'éclairement. Ces facteurs influent d'ailleurs différemment suivant qu'ils interviennent pendant la période de faible activité spermatogénétique ou pendant la spermiogenèse.

Pendant la vie ralentie (de novembre à avril), période normale de jeûne pour l'espèce, une alimentation très abondante entraîne une diminution nette de la durée de la phase de multiplication des spermatogonies primaires qui est normalement de six mois. Ces divisions se déroulent en moins de deux mois lorsque la nourriture est distribuée tous les deux jours et en quatre mois si elle ne l'est plus que tous les dix jours. En revanche un rythme mensuel d'alimentation ne modifie pas la durée de cette phase par rapport au cycle normal. La suite de la gamétogenèse, de la multiplication des spermatogonies secondaires à la maturation des spermatozoïdes, apparaît aussi comme tributaire des facteurs physiques associés à la fréquence d'alimentation (fig. 1). L'association d'un taux d'humidité de 90 p. 100 et d'un éclairement de 1000 lux avec photopériode $12 \mathrm{~L} / 12 \mathrm{~N}$ stimule la spermatogenèse quelle que soit la fréquence d'alimentation. Le rythme d'climentation devient alors limitant : seule la spermiogenèse des mâles nourris tous les deux jours se déroule au même rythme que celle des témoins entre avril et juillet. En atmosphère sèche ( 35 p. 100 d'humidité) la multiplication des spermatogonies I est accélérée comme dans les lots précédents si les animaux sont nourris tous les deux ou tous les dix jours, mais les divisions et les maturations qui suivent cette phase se font bien plus lentement. Ce retard s'aggrave si les animaux sont maintenus à l'obscurité pendant toute la durée de l'expérience.

Pendant la vie active de l'espèce (d'avril à octobre), le facteur nutritionnel reste prépondérant (fig. 2). Quelles que soient les conditions d'humidité et d'éclairement, la spermatogenèse des mâles nourris seulement une fois par mois est profondément perturbée. Les pycnoses des gonies II deviennent quantitativement très importantes deux mois après le début de l'expérience et la spermiogenèse est très en retard par rapport au cycle normal. La mortalité de ce groupe ayant été élevée, il n'est pas possible de conclure à l'existence ou non d'une inhibition de la gamétogenèse due à une sousalimentation. Les facteurs humidité et éclairement modifient de façon variable la spermatogenèse des mâles convenablement alimentés : il s'agit soit de dégénérescences cellulaires, quantitativement et qualitativement plus ou moins importantes, soit 
de retards par rapport au cycle spermatogénétique normal mais en aucun cas la spermiogenèse n'est inhibée : en août, les mâles de tous les lots subissent la spermiation.

L'ensemble de ces expériences n'ayant porté que sur un seul cycle sexuel, il n'est pas possible d'affirmer que le maintien des mâles dans des conditions de vie active (nourriture abondante, taux d'humidité ambiant élevé, éclairement important avec photopériode) supprimerait systématiquement la phase de faible activité spermatogénétique.

\section{Conclusion.}

En novembre, la cessation presque totale des pluies et la chute du degré hygrométrique de l'air qui poussent les $N$. occidentalis à s'enfouir, et la diminution de la quantité de proies accessibles aux crapauds après leur enfouissement, induisent une phase de faible activité spermatogénétique qui dure jusqu'en avril. De novembre à janvier, les testicules, très petits, ne contiennent que des spermatogonies primaires

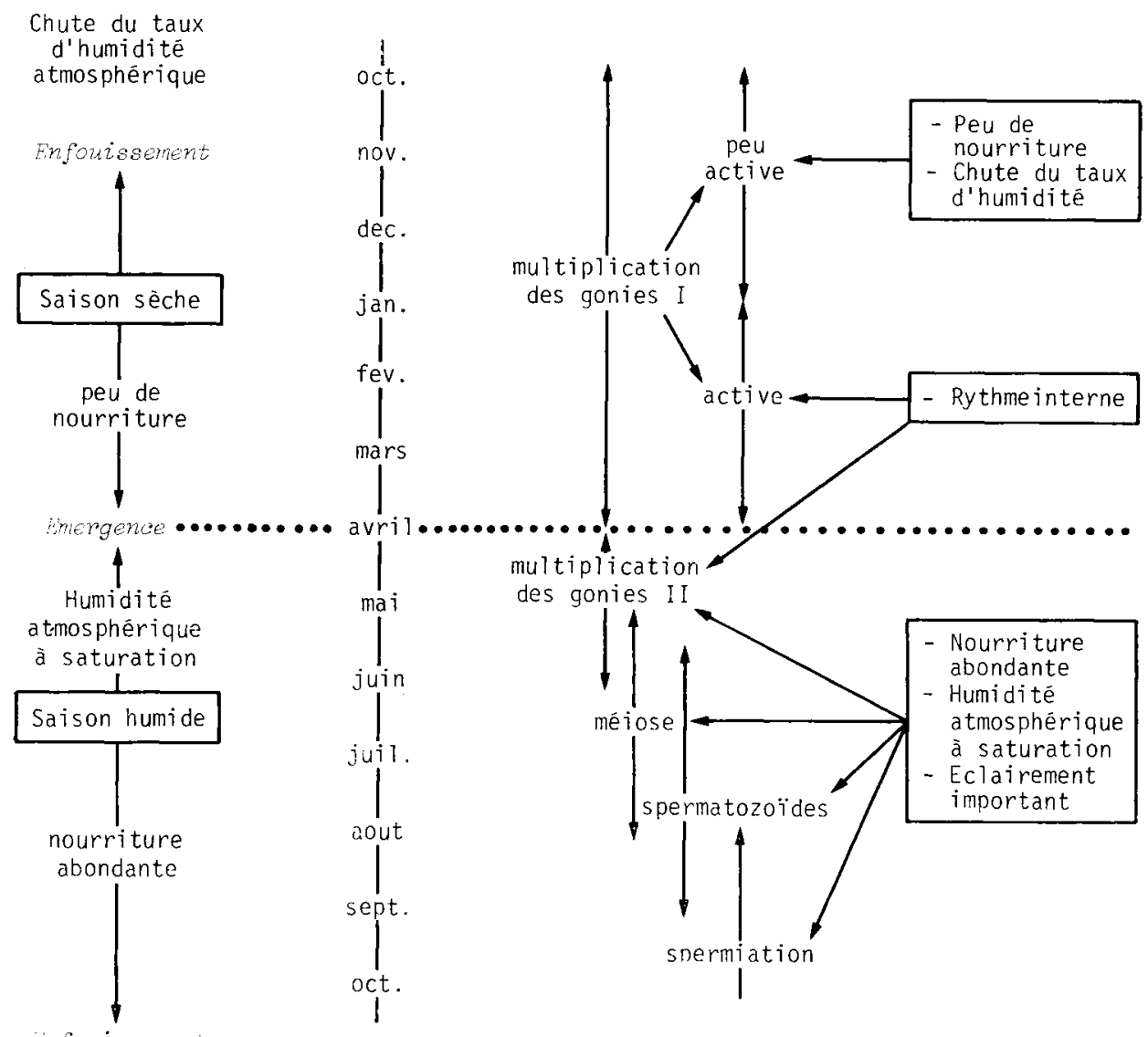

FIG. 4. - Schéma de régulation du cycle spermatogénétique chez N. occidentalis. 
qui se divisent très peu. Si dès novembre des crapauds élevés en atmosphère très humide sont nourris abondamment, cette première phase peut être totalement supprimée. La multiplication des spermatogonies primaires commence alors en novembre ef dure moins de deux mois ; la suite de la spermatogenèse, si les conditions d'élevage restent identiques, se déroule normalement et en avance par rapport au cycle normal (fig. 4).

En janvier, alors que les conditions climatiques et nutritionnelles du biotope sont restées les mêmes depuis novembre, l'activité de division des spermatogonies primaires s'intensifie pour s'achever vers le mois d'avril ; les gonades sont toujours au minimum de leur développement. Cette reprise de l'activité mitotique semble donc être dépendante d'un rythme interne (fig. 4).

En avril, le retour à des conditions de vie favorables (humidité de l'air élevée, nourriture très abondante) pour les $N$. occidentalis entraîne rapidement la croissance pondérale des testicules mais semble sans effet, en soi, sur le déclenchement de la seconde poussée goniale. Plus tard, la multiplication des spermatogonies II étant en cours, seuls les mâles nourris abondamment, élevés en atmosphère humide et soumis à un éclairement important subissent une spermiogenèse normale (fig. 4).

La régulation de l'activité gamétogénétique des mâles de Nectophrynoides occidentalis pourrait dépendre d'une stimulation endogène en janvier et avril au moment des poussées goniales. Pendant toute la durée de la spermiogenèse, les facteurs du milieu - principalement l'alimentation et le taux d'humidité atmosphérique semblent déterminer le bon déroulement de la gamétogenèse.

Reçu en décembre 1976.

Accepté en février 1977.

Remerciements. - Ce travail a bénéficié d'une aide du CNRS dans le cadre de l'ATP, Physiologie écologique (contrat no 449915).

\section{Références}

ANGEL F., LAMOTTE M., 1944. Un crapaud vivipare d'Afrique occidentale, Nectophrynoides occidentalis (Angel). Ann. Sci. nat. Zool., 10, 63-89.

ANGEL F., LAMOTTE M., 1948. Nouvelles observations sur Nectophrynoides occidentalis. Remarques sur le genre Nectophrynoides. Ann. Sci. nat. Zool., 10, 115-147.

BILLARD R., SOLARI A., ESCAFFRE A. M., 1974. Méthode d'analyse quantitative de la spermatogenèse des poissons Téléostéens. Ann. Biol. anim. Bioch. Biophys., 14, 87-104.

CRESPO E. G., CEI J. M., 1971. L'activité spermatogénétique saisonnière de Rana iberica. Arqu. Mus. Bocoge Port., 3, 37-50.

GALGANO M., 1935. Interno all'influenza del clima sull spermatogenesi di Rana esculenta. Arch. ifal. Anat. Embriol., 35, 5-11.

GAVAUD J., 1975. Ełude expérimentale du rôle des facteurs externes sur la spermatogenèse et l'activité endocrine du testicule de Nectophrynoides occidentalis Angel. Publ. Lab. Zool. E. N. S., 6, 1-99.

GAVAUD J., 1976. La gamétogenèse du mâle de Nectophrynoides occidentalis Angel (Amphibien Anoure vivipare). I. Elude quantitative au cours du cycle annuel chez l'adulte. Ann. Biol. anim. Bioch. Biophys., 16, 1-12.

GUISTI L., HOUSSAY B. A., 1923. Altérations cutanées et génitales produites par l'extirpation de I'hypophyse ou par lésion du cerveau. C. R. Soc. Biol. Paris, 91, 313. 
LAMOTTE M., 1959. Observations sur les populations naturelles de Nectophrynoides occidentalis Angel. Bull. Biol. Fr. et Belg., 93, 314-355.

LAMOTTE M., 1972. Bilan énergétique de la croissance du mâle de Necfophrynoides occidentalis Angel, Amphibien Anoure. C. R. Acad. Sc. Paris, série D, 274, 2074-2076.

OORDT (Van) P. G. W., 1956. Regulation of the spermatogenefic cycle in the common frog Rana temporaria. G. W. van Der Wiel and Co ; Arnhem, 116 p.

OORDT (Van) P. G. W., 1960. The influence of internal and external factors in the regulation of the spermatogenetic cycle in Amphibia. Symp. Zool. Soc. Lond., 2, 29-52.

SOLARI A., 1973. Etude quantitative d'organes ou de tissus. I. Méthodes d'estimation des volumes. Ann. Biol. anim. Bioch. Biophys., 13, 247-265.

WERNER J. K., 1969. Temperature-photoperiod effects on spermatogenesis in the salamander. Plethodon cinereus. Copeia, 3, 592-601.

WITSCHI E., 1924. Die Entwicklung der Keimzellen der Rana temporaria L. I. Urkeimzellen und Spermatogenese. Z. ZII. u. Gewebelehre, 1, 523.

ZUBER-VOGELI M., XAVIER F., 1965. La spermatogenèse du mâle de Nectophrynoides occidentalis Angel, au cours du cycle annuel. Bull. Soc. Zool. fr., 90, 261-267. 